\title{
TERAPÊUTICA MEDICAMENTOSA PRESCRITA CONFORME QUEIXA INICIAL E SINAL/SINTOMA A USUÁRIOS DO CENTRO DE ATENÇÃO PSICOSSOCIAL INFANTO-JUVENIL
}

DOI: 10.22289/2446-922X.V7N2A6

\section{RESUMO}

\author{
Nevoni Goretti Damo ${ }^{1}$ \\ Samira Raquel de Farias Wackernagel \\ Ana Beatriz dos Santos \\ Arthur Mandalis Sônego \\ Wallace Mees
}

A prescrição e o consumo de medicamentos psiquiátricos por crianças e adolescentes é um dos motivos de preocupação para a sociedade brasileira. Estes medicamentos, prescritos para o tratamento de transtornos mentais e sofrimento psíquico, são recursos terapêuticos comumente utilizados no atendimento a usuários de Centros de Atenção Psicossocial Infanto-juvenil (CAPSi). O objetivo deste estudo foi descrever a terapêutica prescrita conforme a queixa inicial e sinais/sintomas de usuários do CAPSi de Blumenau. Para tal, foram analisados e coletados dados de prontuários de crianças e adolescentes, de ambos os sexos, de agosto a dezembro de 2018 . Em relação aos resultados encontrados, o sexo masculino foi o mais frequente, $58,3 \%(n=28)$ e a faixa etária mais prevalente foi entre 13 anos a 18 anos de idade $75 \%(n=36)$. O principal medicamento prescrito como forma de tratamento foi a Risperidona $22,10 \%(n=21)$. As queixas iniciais mais comuns foram agressividade e ansiedade $8,88 \%(n=08)$. O sinal/sintoma mais comum foi a ansiedade $13,40 \%(n=13)$. De acordo com a literatura, as substâncias mais prescritas encontradas nesta pesquisa podem beneficiar o tratamento das queixas e dos sinais/sintomas das crianças e adolescentes com sofrimento psíquico. As substâncias Risperidona, Cloridrato de Fluoxetina e Oxalato de Escitalopram são as principais substâncias prescritas aos usuários do CAPSi. Estas substâncias prescritas por especialistas e utilizadas de maneira racional podem beneficiar 0 tratamento dos usuários. Desta maneira, é fundamental destacar a importância de ampliar a discussão sobre prescrições de medicamentos não disponíveis no SUS, pois o principal medicamento prescrito não consta na REMUME.

Palavras-chave: Medicalização; Saúde Mental; Uso Racional de Medicamentos.

\section{DRUG THERAPEUTIC PRESCRIBED ACCORDING TO INITIAL COMPLAINT AND SIGN / SYMPTOM TO USERS OF THE INFANTO-JUVENILE PSYCHOSOCIAL CARE CENTER}

\section{ABSTRACT}

\footnotetext{
${ }^{1}$ Endereço eletrônico de contato: nevoni@furb.br

Recebido em 15/04/2021. Aprovado pelo conselho editorial para publicação em 19/07/2021.
}

Rev. Psicol Saúde e Debate. Jul., 2021:7(1): 83-96. 
The prescription and consumption of psychiatric drugs by children and adolescents is one of the reasons of concern for Brazilian society. These medications, prescribed for the treatment of mental disorders and psychological distress, are therapeutic resources commonly used to assist users of Psychosocial Care Centers (CAPSi). The aim of this study was to describe the therapy prescribed according to the initial complaint and signs / symptoms of CAPSi users. To this end, data from medical records of children and adolescents, of both sexes, were analyzed and collected between August and December 2018. Regarding the results found, the male gender was the most frequent, $58.3 \%(n=28)$ and the most prevalent age group was between 13 years and 18 years of age $75 \%$ $(n=36)$. The main medication prescribed as a form of treatment was Risperidone $22.10 \%(n=21)$. The most common initial complaints were aggressiveness and anxiety $8.88 \%(n=08)$. The most common sign / symptom was $13.40 \%$ anxiety $(n=13)$. According to the literature, the most prescribed substances found in this research can benefit the treatment of complaints and signs / symptoms of children and adolescents being treated at CAPSi. Therefore, the substances Risperidone, Fluoxetine Hydrochloride and Escitalopram Oxalate are the main substances prescribed to CAPSi users. These substances prescribed by specialists and used in a rational manner can benefit the treatment of users. Thus, it is essential to highlight the importance of expanding the discussion on drug prescriptions not available in SUS, as the main drug prescribed is not included in REMUME.

Keywords: Medicalization; Mental health; Rational Use of Medicines.

\section{MEDICAMENTOS TERAPÉUTICOS PRESCRITOS SEGÚN DENUNCIA INICIAL Y SIGNO / SÍNTOMA A USUARIOS DEL CENTRO DE ATENCIÓN PSICOSOCIAL INFANTO-JUVENIL}

\section{RESUMEN}

La prescripción y consumo de psicofármacos por niños y adolescentes es uno de los motivos de preocupación de la sociedad brasileña. Estos medicamentos, recetados para el tratamiento de trastornos mentales y angustia psicológica, son recursos terapéuticos comúnmente utilizados para ayudar a los usuarios de los Centros de Atención Psicosocial (CAPSi). El objetivo de este estudio fue describir la terapia prescrita de acuerdo con la queja inicial y los signos / síntomas de los usuarios de CAPSi. Para ello, se analizaron y recolectaron datos de las historias clínicas de niños y adolescentes, de ambos sexos, entre agosto y diciembre de 2018. En cuanto a los resultados encontrados, el género masculino fue el más frecuente, $58,3 \%(n=28)$ y el grupo de edad más prevalente fue entre 13 y 18 años $75 \%(n=36)$ El principal medicamento prescrito como forma de tratamiento fue Risperidona $22,10 \%(n=21)$. Las quejas iniciales más frecuentes fueron agresividad y ansiedad $8,88 \%(n=08)$. El signo / síntoma más común fue $13,40 \%$ de ansiedad $(n=13)$. Según la literatura, las sustancias más prescritas encontradas en esta investigación pueden beneficiar el tratamiento de quejas y signos / síntomas de niños y adolescentes que están siendo tratados en CAPSi. Por tanto, las sustancias risperidona, hidrocloruro de fluoxetina y oxalato de escitalopram son las principales sustancias prescritas a los usuarios de CAPSi. Estas sustancias prescritas por especialistas y utilizadas de manera racional pueden beneficiar el tratamiento de los usuarios. Por ello, es fundamental resaltar la importancia de ampliar la discusión sobre prescripciones de medicamentos no disponibles en el SUS, ya que el principal fármaco prescrito no está incluido en REMUME.

Palabras clave: Medicalización; Salud Mental; Uso Racional de Medicamentos. 


\section{INTRODUÇÃO}

No Brasil, no que se refere à Saúde Mental Infanto-Juvenil há dois marcos institucionais importantes para os direitos da criança e do adolescente, a implantação do Sistema Único de Saúde (SUS) (Lei no 8.080/1990) e a Lei ํo 10.216/2001, referente à Reforma Psiquiátrica Brasileira. O SUS garante o atendimento à população infanto-juvenil e suas famílias conforme os princípios da universalidade, da integralidade, da equidade e o acesso à saúde; e a Reforma Psiquiátrica Brasileira visa o atendimento das pessoas com sofrimento psíquico, para que este ocorra em liberdade e em seus territórios de referência (Brasil, 2001).

Assim, para o atendimento de crianças e adolescentes, a Portaria no 336 do Ministério da Saúde (MS), regulamentou em 2002 o CAPSi, serviço de atenção diária destinado ao atendimento de crianças e adolescentes com graves prejuízos psíquicos (Ministério da Saúde, 2005). Este dispositivo para a Saúde Mental Infanto-Juvenil, que surgiu quase doze anos depois de promulgado - Estatuto da Criança e do Adolescente (ECA), é indicado para municípios ou regiões com população acima de 70 mil habitantes, conforme Ministério da Saúde (2015).

O CAPSi atende crianças e adolescentes autistas, com psicoses, neuroses graves e todos aqueles impossibilitados de manter ou estabelecer laços sociais em decorrência do seu comprometimento psíquico. A partir desta lógica, ampliaram-se as possibilidades do tratamento propondo-se a intervenção precoce, por meio de parcerias com a rede de Saúde, da Educação e da Assistência Social, com ações destinadas ao cuidado da população infanto-juvenil por meio de grupos terapêuticos, atendimentos individuais, consultas médicas e uso de medicamentos prescritos por médicos psiquiatras da infância e adolescência (Ministério da Saúde, 2004).

No que se refere ao tratamento medicamentoso, segundo Araújo (2017), as substâncias psicoativas são o principal grupo de escolha dos psiquiatras como recurso terapêutico oferecido pelo serviço de saúde. Estas substâncias, prescritas para o tratamento de transtornos mentais e sofrimento psíquico, atuam principalmente no sistema nervoso central, alterando a função cerebral, sendo seu o consumo motivo de diversos estudos e de preocupação na sociedade brasileira, principalmente quando utilizadas por crianças e adolescentes.

A Organização Mundial da Saúde (OMS), defende que o Uso Racional de Medicamentos (URM) está presente nos diferentes níveis da Assistência Farmacêutica, promovendo ações de promoção, proteção e recuperação da saúde, com foco no acesso e uso racional dos medicamentos. No Brasil, a Relação Nacional de Medicamentos Essenciais (RENAME) e a Relação Municipal de Medicamentos Essenciais (REMUME) possuem como principais objetivos orientar e promover o acesso e o uso seguro e racional dos medicamentos.

É necessário contextualizar e refletir sobre a prescrição da medicação relacionada ao fracasso das outras abordagens às expectativas dos pais e/ou pressão da escola (Arantes, 2014). Isto se deve ao fato de que em algumas situações, problemas que não eram considerados de ordem Rev. Psicol Saúde e Debate. Jul., 2021:7(1): 83-96. 
médica passaram a ser vistos e tratados como problemas médicos, resultando no processo da medicalização (Brzozowski \& Caponi, 2013).

Sendo assim, algumas das substâncias, prescritas aos usuários do CAPSi, são dispensadas de acordo com a disponibilidade dos medicamentos na lista da RENAME e/ou REMUME (Yamauti et al., 2017). Desta maneira, contribui para que os usuários tenham acesso aos medicamentos e proporcionar melhores tratamentos para as crianças e os adolescentes atendidos melhorando a qualidade de vida destes e garantindo terapias medicamentosas mais adequadas.

Diante da relevância do tema, tem-se como objetivo neste estudo descrever a terapêutica prescrita conforme a queixa inicial e sinais/sintomas; identificar os principais medicamentos prescritos; relacionar os medicamentos com as queixas iniciais e/ou sinais/sintomas; analisar a porcentagem de medicamentos prescritos que pertencem na RENAME e/ou na REMUME.

\section{MÉTODOS}

Trata-se de um estudo descritivo, retrospectivo de abordagem quantitativa com dados obtidos de prontuários físicos e eletrônicos de usuários assistidos pela equipe de profissionais da saúde do CAPSi. Este trabalho teve, inicialmente, como sujeitos da pesquisa 107 crianças e adolescentes assistidos no serviço. A coleta e análise dos dados foi realizada de agosto a dezembro do ano de 2018, durante as atividades do PET-Saúde/GraduaSUS no CAPSi de Blumenau/SC.

Os dados foram coletados dos prontuários com prescrição de medicamentos para os usuários atendidos no CAPSi, após assinatura do Termo de Consentimento Livre e Esclarecido (TCLE) pelos seus responsáveis. O estudo teve como critério de inclusão ter prescrição medicamentosa no último atendimento médico realizado no CAPSi e como critério de exclusão não ter prescrição medicamentosa no último atendimento médico realizado. A coleta dos dados foi realizada em 44,85\% $(n=48)$ da amostra inicial. O principal motivo de não ter alcançado a amostra desejada $(n=107)$ foram as recusas ou ausências dos responsáveis das crianças e adolescentes assistidos no serviço para concordarem com o estudo e assinarem o TCLE. O estudo foi aprovado pelo Comitê de Ética em humanos sob o parecer ํㅜㄴ 2.731.093.

Foram coletados dados dos prontuários físicos e eletrônicos, tais como: sexo, idade, queixa inicial, sinais/sintomas e medicação(ões) prescritas durante a última consulta médica. $O$ instrumento para a coleta foi do tipo checklist desenvolvido para a pesquisa. Os dados foram registrados por código numéricos para garantir o sigilo do participante, armazenados no Excel e posteriormente apresentados na forma de tabelas. 


\section{RESULTADOS E DISCUSSÃO}

A Organização Mundial de Saúde (OMS) (1985) expõe que, para o URM, é preciso estabelecer a necessidade do uso do medicamento e em seguida que se receite o medicamento apropriado, na forma farmacêutica, doses e período de duração do tratamento adequado; que esteja disponível de modo oportuno, a preço acessível, e que responda sempre aos critérios de qualidade exigidos; que se dispense em condições adequadas, com a necessária orientação e responsabilidade. A prescrição de medicamentos psicotrópicos para crianças e adolescentes necessita de avaliação das substâncias utilizadas (Araújo, 2017).

Da amostra coletada 58,3\% $(n=28)$ dos usuários são sexo masculino e 41,7\% (n=20) do sexo feminino. As idades encontradas foram de 06 a 18 anos, com a faixa etária prevalente de 13 anos a 18 anos de idade $75 \%(n=36)$. Nos estudos referentes ao CAPSi de Leitão et al. (2020) e Silvia et al. (2020), as idades mais frequentes encontradas são semelhantes às apresentadas neste estudo e o sexo predominante também foi o masculino.

De acordo com a Organização Mundial da Saúde (WHO) (1986), define a adolescência como a faixa etária de dez e dezenove anos de idade. Neste estudo os adolescentes são os principais participantes, tendo um percentual de prevalência $75 \%$. O período da adolescência é marcado, principalmente, pela busca de autonomia sobre as decisões, emoções e ações vivenciadas. É um período em que os seres humanos exploram mais intensamente sua identidade sexual e de gênero. Em alguns casos, as experiências possibilitam uma maior exposição às violências, aos comportamentos de riscos, o uso drogas lícitas e/ou ilícitas, suscetibilidade às infecções sexualmente transmissíveis (ISTs). Atualmente, os problemas de Saúde Mental, principalmente a depressão, agressividade e ansiedade, manifestam-se gradativamente na fase da adolescência (Araújo, 2017).

Foram encontradas 33 queixas iniciais que se repetiram ou não, e estão descritas na Tabela 1. A maior parte dos usuários em tratamento no CAPSi apresentou 1 queixa 23,3\% ( $n=21)$, 2 queixas $16,6 \%(n=15)$, 3 queixas $9,9 \%(n=09)$ e 4 queixas iniciais 3,3\% (n=03). As queixas iniciais mais frequentes foram agressividade e ansiedade, seguidas de automutilação, tentativa de suicídio e transtorno de comportamento.

Tabela 1- Queixas Iniciais apresentadas no acolhimento e suas frequências:

\begin{tabular}{llll}
\hline & QUEIXA INICIAL & (n) & $\%$ \\
\hline Agressividade & 08 & 8,88 \\
Ansiedade & 08 & 8,88 \\
Automutilação & 06 & 6,66 \\
Tentativa de suicídio & 06 & 6,66 \\
\hline
\end{tabular}

Rev. Psicol Saúde e Debate. Jul., 2021:7(1): 83-96. 


\begin{tabular}{|c|c|c|}
\hline Transtorno de comportamento & 06 & 6,66 \\
\hline Agitação & 04 & 4,44 \\
\hline Alucinações & 04 & 4,44 \\
\hline Depressão & 04 & 4,44 \\
\hline Ideação suicida & 04 & 4,44 \\
\hline Tristeza & 04 & 4,44 \\
\hline Anedonia & 02 & 2,22 \\
\hline Autismo & 02 & 2,22 \\
\hline Compulsão alimentar & 02 & 2,22 \\
\hline Conflitos familiares & 02 & 2,22 \\
\hline Hiperatividade & 02 & 2,22 \\
\hline Isolamento & 02 & 2,22 \\
\hline Substâncias psicoativas & 02 & 2,22 \\
\hline Transtorno de humor & 02 & 2,22 \\
\hline Abstinência & 01 & 1,11 \\
\hline Bullying & 01 & 1,11 \\
\hline Conduta inadequada & 01 & 1,11 \\
\hline Conduta sexualizada & 01 & 1,11 \\
\hline Dificuldade de relacionamento & 01 & 1,11 \\
\hline Fobia & 01 & 1,11 \\
\hline Insônia & 01 & 1,11 \\
\hline Irritabilidade & 01 & 1,11 \\
\hline Medo & 01 & 1,11 \\
\hline Movimentos e sons involuntários & 01 & 1,11 \\
\hline Problemas escolares & 01 & 1,11 \\
\hline Sintomas depressivos & 01 & 1,11 \\
\hline TAD & 01 & 1,11 \\
\hline TDAH & 01 & 1,11 \\
\hline Zoofilia & 01 & 1,11 \\
\hline
\end{tabular}

FONTE: Autores (2020).

As queixas iniciais eram identificadas e descritas nos prontuários, pela equipe multiprofissional (assistente social, fonoaudiólogo, psicólogos, terapeutas ocupacionais, enfermeiros, técnicos de enfermagem) do serviço de Saúde Mental, no momento do acolhimento do usuário e de sua família. Moreira et al., (2018) descreve: 
"que um dos propósitos do acolhimento é o seu intuito de iniciar uma avaliação do caso, assim, podendo identificar as principais queixas e possíveis fatores de risco e o contexto sociocultural do usuário, contribuindo para uma possível avaliação da Saúde Mental".

As queixas iniciais mais frequentes encontradas nesta pesquisa são motivos de discussão nas literaturas infanto-juvenil. A agressividade e a ansiedade são consideradas queixas frequentes da infância e adolescência podendo-se relacionar estas queixas com questões que envolvam as adaptações em que os indivíduos são submetidos durante este período e não sabem como agir, e, consequentemente expressam estes sentimentos agressivos, ansiosos ou até mesmo violência autoprovocada - automutilação, tentativa de suicídio e suicídio (Araújo, 2017; Leitão et al., 2020; Silva et al., 2017).

O Ministério da Saúde (2010) expõe que os problemas com a violência autoprovocada na população infanto-juvenil no Brasil tem se tornado questão de saúde pública, devido a correlação deste tema com a automutilação, a tentativa de suicídio e o suicídio consumado. O suicídio, segundo a OMS (2018), é a segunda principal causa de morte entre jovens, considerado um fenômeno complexo e multifatorial. O índice de casos de suicídio na população jovem chama atenção. Os suicídios podem ser evitados, portanto, faz-se necessário fornecer subsídios como forma de prevenção aos adolescentes que se encontram em situações de vulnerabilidade e/ou com comportamentos de violências autoprovocadas (OMS, 2018).

Nos 48 prontuários analisados para esta pesquisa, foram encontrados 32 sinais/sintomas que se repetiram ou não (Tabela 2). A maioria dos usuários em tratamento no CAPSi apresentaram durante as consultas médicas 1 sinal/sintoma 17,5\% $(n=17), 2$ sinais/sintomas $17,5 \%(n=17), 3$ sinais/sintomas $7,2 \%(n=07)$ e 4 sinais/sintomas $4,23 \%(n=04)$. Os sinais/sintomas mais frequentes foram ansiedade e depressão, seguidos de agressividade. Os sinais e sintomas são importantes, pois possibilitam ao profissional reconhecer alterações físicas, mentais, fenômenos, formular o diagnóstico e a terapêutica a ser utilizada (Dalgalarrondo, 2018).

Tabela 2- Sinais/sintomas e suas frequências.

\begin{tabular}{llll}
\hline & Sinais e sintomas & (n) & $\%$ \\
\hline Ansiedade & 13 & 13,40 \\
Depressão & 12 & 12,37 \\
Agressividade & 07 & 7,22 \\
Automutilação & 06 & 6,19 \\
Conflitos familiares & 04 & 4,12 \\
Déficit intelectual & 04 & 4,12 \\
Transtorno afetivo bipolar & 04 & 4,12 \\
Distúrbio de conduta & 03 & 3,09 \\
\hline
\end{tabular}




\begin{tabular}{|c|c|c|}
\hline Irritabilidade & 03 & 3,09 \\
\hline Substâncias psicoativas & 03 & 3,09 \\
\hline Transtorno de comportamento & 03 & 3,09 \\
\hline Transtorno de humor & 03 & 3,09 \\
\hline Autismo & 02 & 2,06 \\
\hline Episódios depressivos & 02 & 2,06 \\
\hline Isolamentos & 02 & 2,06 \\
\hline TOC & 02 & 2,06 \\
\hline Transtorno ansioso & 02 & 2,06 \\
\hline Agitação & 01 & 1,03 \\
\hline Agitação psicomotora & 01 & 1,03 \\
\hline Alucinação & 01 & 1,03 \\
\hline Autoagressão & 01 & 1,03 \\
\hline Bulimia & 01 & 1,03 \\
\hline Compulsão alimentar & 01 & 1,03 \\
\hline Fobia & 01 & 1,03 \\
\hline Hiper sexualidade & 01 & 1,03 \\
\hline Ideação suicida & 01 & 1,03 \\
\hline Síndrome de La tourette & 01 & 1,03 \\
\hline Retardo mental leve & 01 & 1,03 \\
\hline Transtorno de Déficit de Atenção com Hiperatividade & 01 & 1,03 \\
\hline Tentativa de suicídio & 01 & 1,03 \\
\hline Transtorno bipolar generalizado & 01 & 1,03 \\
\hline Tristeza & 01 & 1,03 \\
\hline
\end{tabular}

FONTE: Autores (2020).

Os sinais/sintomas identificados por médicos especialistas durante as consultas são fundamentais para tratamento adequado. Como cita Leitão et al. (2020), "para uma prescrição de medicamentos, principalmente psiquiátricos, para crianças e adolescentes deve-se levar em consideração as hipóteses diagnósticas e/ou sinal/ sintoma".

Em relação aos sinais/sintomas encontrados, a depressão é um dos sinais/sintomas mais frequentes nos usuários do CAPSi. A depressão é um transtorno mental frequente, estima-se que aproximadamente 300 milhões de pessoas, de todas as idades, no mundo sofram com esse transtorno. A depressão e outras condições de saúde mental são consideradas problemas de saúde pública no mundo. O diagnóstico de depressão e outros transtornos são muitas vezes diagnosticados de maneira inadequada, podendo resultar em intervenções desnecessárias e 
aumento de casos de suicídio, pois estes dois transtornos estão relacionados (OMS, 2018). Diante disso, pode-se destacar a importância de discutir este tema na formação dos profissionais de saúde.

A formulação de diagnóstico de qualidade exige procedimentos de avaliação específicos. Entretanto, sabe-se que mesmo considerando os avanços dos métodos de diagnósticos ocorridos na última década em relação às estratégias de definição e avaliação da severidade das patologias mentais infanto-juvenil, ainda se tem muito a evoluir no seu estudo e tratamento (Couto, Duarte \& Delgado, 2008). Na sociedade atual o diagnóstico se sustenta no processo de medicalização o que proporciona aumento na prescrição e uso de medicamentos.

O sociólogo Peter Conrad (2007) apresenta o seguinte conceito: "medicalização descreve um processo pelo qual problemas não médicos passam a ser definidos e tratados como problemas médicos, frequentemente em termos de doenças ou transtornos". A psicopatologia refere-se à medicalização mais especificamente à transformação de comportamentos desviantes em doenças ou transtornos mentais, implicando a ação do controle e poder médico (Dalgalarrondo, 2018).

Nesse estudo 19 medicamentos pertencentes a 6 classes farmacológicas diferentes foram prescritos a crianças e adolescentes. Os medicamentos encontrados e suas frequências estão descritas na Tabela 3. Dos 19 medicamentos prescritos, constam 47,36\% ( $n=9)$ na lista de medicamentos essenciais RENAME e 52,64\% $(n=10)$ na REMUME.

Tabela 3 - Presenças $(\mathrm{S})$ ou não $(\mathrm{N})$ na lista de medicamentos essenciais e suas frequências nas prescrições.

\begin{tabular}{ccccc}
\hline MEDICAMENTOS & REMUME & RENAME & (n) & $\%$ \\
\hline ANSIOLÍTICOS & & & & \\
Clonazepam & $\mathrm{S}$ & $\mathrm{N}$ & 02 & 2,10 \\
ANTICOLINÉRGICOS & & $\mathrm{N}$ & 02 & 2,10 \\
Biperideno & $\mathrm{S}$ & & & \\
ANTIDEPRESSIVOS & & $\mathrm{S}$ & 17 & 17,89 \\
Cloridrato de Fluoxetina & $\mathrm{S}$ & $\mathrm{N}$ & 02 & 2,10 \\
Cloridrato de Imipramina & $\mathrm{S}$ & $\mathrm{N}$ & 05 & 5,26 \\
Cloridrato de Sertralina & $\mathrm{N}$ & $\mathrm{N}$ & 13 & 13,68 \\
Oxalato de Escitalopram & $\mathrm{N}$ & & & \\
ANTIPSICÓTICOS & & $\mathrm{N}$ & 01 & 1,05 \\
Aripiprazol & $\mathrm{N}$ & $\mathrm{S}$ & 04 & 4,21 \\
Clorpromazina & $\mathrm{S}$ & $\mathrm{S}$ & 01 & 1,05 \\
\hline Hemifumarato de Quetiapina & $\mathrm{N}$ & $\mathrm{N}$ & 03 & 3,15 \\
Levomepromazina & $\mathrm{S}$ & $\mathrm{N}$ & 01 & 1,05 \\
Paliperidona & $\mathrm{N}$ & $\mathrm{N}$ & 01 & 1,05 \\
Pimozida & $\mathrm{N}$ & $\mathrm{S}$ & 21 & 22,10 \\
Risperidona & $\mathrm{N}$ & $\mathrm{N}$ & 01 & 1,05 \\
Tioridazida & $\mathrm{N}$ & $\mathrm{S}$ & 05 & 5,26 \\
\hline Haloperidol & $\mathrm{S}$ & & & \\
\hline
\end{tabular}




\begin{tabular}{ccccc}
\hline $\begin{array}{c}\text { ESTABILIZANTES DE HUMOR E } \\
\text { ANTICONVULSIVANTES }\end{array}$ & & & & \\
Ácido Valpróico & $\mathrm{S}$ & $\mathrm{S}$ & 05 & 5,26 \\
Carbonato de Lítio & $\mathrm{S}$ & $\mathrm{S}$ & 05 & 5,26 \\
$\quad$ Topiramato & $\mathrm{N}$ & $\mathrm{S}$ & 03 & 3,15 \\
\hline $\begin{array}{c}\text { ESTIMULANTE DO SISTEMA } \\
\text { NERVOSO CENTRAL }\end{array}$ & & & & \\
Metilfenidato & $\mathrm{S}$ & $\mathrm{S}$ & 05 & 5,26 \\
\hline
\end{tabular}

Legenda: N: NÃO S: SIM

FONTE: Autores (2020).

Os medicamentos mais prescritos foram o antipsicótico Risperidona e o antidepressivo Cloridrato de Fluoxetina, seguidos do antidepressivo Oxalato de Escitalopram. Dos usuários em tratamento no CAPSi teve em seu prontuário a prescrição médica de 2 medicamentos $22,1 \%(n=21)$, 1 medicamento 16,8\% ( $n=16)$, 3 medicamentos $7,4 \%(n=07)$ e 4 medicamentos $4,2 \%(n=04)$.

As duas principais classes de medicamentos prescritos encontrados nesse estudo foram os antipsicóticos e antidepressivos. A Risperidona, antipsicótico atípico, foi o medicamento mais prescrito como forma de tratamento medicamentoso, seguida dos antidepressivos. Estas são as principais classes de medicamentos encontrados prescritos aos usuários em tratamento no CAPSi (Leitão et. al, 2020; Silva et. al, 2020).

Os antipsicóticos, em especial os antipsicóticos atípicos (Aripiprazol, Hemifumarato de Quetiapina, Risperidona, Paliperidona, Pimozida) são os fármacos mais usados e aprovados para o tratamento de crianças e adolescentes com Transtornos do Espectro do Autismo (TEA), quadros de psicoses, quadros de agressividade, ansiedade e depressão que necessitam de associação de medicamentos com classes farmacológicas distintas. A Risperidona, escolha mais frequente dos profissionais da área médica, por apresentar maior segurança e efetividade no tratamento de crianças e adolescentes com os sinais/sintomas encontrados nesta pesquisa (Brasil \& Filho, 2000; Silva, 2017; Teixeira et al., 2013).

Com relação aos antidepressivos, estes são prescritos principalmente para o tratamento de transtornos depressivos, de ansiedade e de comportamento. Os antidepressivos pertencentes à classe dos inibidores seletivos da recaptação de serotonina (ISRS), como o Cloridrato de Fluoxetina e Oxalato de Escitalopram, e os antidepressivos tricíclicos (ADTs), classe do Cloridrato de Imipramina, são as principais classes de antidepressivos prescritos como forma de tratamento para crianças e adolescentes. Os antidepressivos da classe dos ISRS são prescritos com maior frequência ao público infanto-juvenil quando comparados aos ADTs, principalmente devido seus efeitos colaterais (Brasil \& Filho, 2000; Silva, 2017).

Embora às vezes seu uso seja incoerente e/ou prescrito sem formulação de hipótese diagnóstica, a medicação é utilizada como tratamento para as crianças e adolescentes no Serviço de Saúde Mental. O uso de medicação na Saúde Mental infanto-juvenil requer um cuidado especial, 
em razão de que estes medicamentos psiquiátricos apresentam diversos eventos adversos e requerem uso controlado (Leitão et. al, 2020; Silva et. al, 2020).

Com relação a RENAME e a REMUME são listas de medicamentos que devem atender às necessidades de saúde prioritárias da população brasileira, listando medicamentos eficazes, seguros e de melhor custo-benefício. Alguns dos medicamentos essenciais incluídos nas listas não contemplam as prioridades na saúde pública, sendo assim, percebe-se a importância de atualizações anuais das listas (Yamauti et al., 2017).

Em relação aos medicamentos presentes nas listas de medicamentos essenciais, RENAME (2020) e REMUME (2019), as porcentagens encontradas no presente estudo chamam a atenção. Dos medicamentos prescritos às crianças e adolescentes, apenas 68,42\% (13) estão presentes nas listas. Visto que, os medicamentos mais prescritos foram o antipsicótico Risperidona e o antidepressivo Oxalato de Escitalopram não se encontravam nas listas para atender as demandas, durante a coleta de dados, gerando impasse, pois, o CAPSi é um dos serviços públicos de saúde em que as prescrições entregues ao público devem constar de medicamentos presentes nas listas de medicamentos essenciais.

O medicamento Risperidona foi incorporado à RENAME somente em 2019, porém não incorporado a REMUME, de acordo com diversos autores entre eles o citado nesse estudo (Teixeira et al., 2013), há alguns anos que este medicamento tem comprovação de eficácia no tratamento de transtornos mentais em crianças e adolescentes. Diante disso, cabe também refletir sobre o acesso versus adesão ao tratamento na atenção a crianças e adolescentes.

A seleção de medicamentos essenciais disponíveis no serviço público de saúde deve ter como base, além das evidências científicas comprovadas, as principais propostas de tratamentos farmacológicos presentes nos protocolos clínicos e diretrizes terapêuticas. A ausência de medicamentos na REMUME pode resultar na aquisição de medicamentos de maneira judicial (Yamauti et al., 2017). Como discutido anteriormente, os medicamentos indicados para o tratamento aos usuários precisam constar nas listas de medicamentos essenciais, sendo um direito dos usuários para terem suas necessidades de saúde atendidas na forma da lei.

Cabe ainda relatar a inexistência de farmácia e do farmacêutico na equipe multiprofissional do CAPSi o que pode dificultar/influenciar o acesso e adesão ao tratamento medicamentoso.

É importante salientar a importância das atividades que envolvam crianças, adolescentes e seus responsáveis para o URM, e que este pode contribuir para o aumento da adesão ao tratamento e uso de medicação dos usuários. Como Silva et al. (2020) destacam em seu estudo, nos CAPSi o tratamento deve ser realizado em conjunto com os responsáveis, visto isso como uma ação importante para a orientação sobre o tratamento, acesso ao serviço e recursos terapêuticos oferecidos. 


\section{CONSIDERAÇÕES FINAIS}

Pode-se concluir que os principais medicamentos prescritos aos usuários do CAPSi, são os que atuam, principalmente, no Sistema Nervoso Central sendo as classes farmacológicas dos antipsicóticos, em especial os atípicos, e os antidepressivos, os ISRS, as mais prescritas. As substâncias Risperidona, Cloridrato de Fluoxetina e Oxalato de Escitalopram são as substâncias mais prescritas aos usuários que apresentaram queixas iniciais e/ou sinais/sintomas, principalmente, de ansiedade, agressividade e depressão. Cabe destacar a necessidade de revisão da REMUME por apresentar medicamentos prescritos não presentes na relação do município, como é o caso da Risperidona e o Oxalato de Escitalopram.

As substâncias psicoativas prescritas por médicos psiquiatras às crianças e aos adolescentes, usuários do CAPSi, quando utilizadas de maneira racional pelos usuários, podem beneficiar o tratamento para as queixas dos sinais/sintomas apresentados. Importante ampliar a discussão sobre prescrições de medicamentos não disponíveis no SUS, tendo em vista que esses medicamentos podem impactar significativamente no cotidiano das famílias.

\section{REFERÊNCIAS}

Arantes, R. L.; Silva, M. M. C.; Araújo, L. O. (2014). Saúde Mental na Infância e Adolescência: Atenção Psicossocial na infância - Florianópolis (SC): Universidade Federal de Santa Catarina, p. 132.

Araújo, S.H.M. (2017). Perfil farmacoterapêutico de adolescentes usuários de um centro de atenção psicossocial álcool e drogas infantojuvenil do estado de Goiás. Dissertação (Mestrado) Universidade Federal de Goiás, Faculdade Farmácia (FF), Programa de Pós-Graduação em Assistência e Avaliação em Saúde, Goiânia, 88p. From: http://repositorio.bc.ufg.br/tede/bitstream/tede/7570/5/Disserta\%c3\%a7\%c3\%a30\%20\%20San dra\%20Hernandez\%20Morais\%20de\%20Ara\%c3\%bajo\%20-\%202017.pdf

Blumenau, Secretaria Municipal de Promoção à Saúde (SEMUS) (2019). Relação Municipal de Medicamentos https://www.blumenau.sc.gov.br/downloads/semus/remume.pdf

Brasil, H. H. A.; Filho, J. F.B. (2000). Psicofarmacoterapia. Rev. Bras. Psiquiatria, 22(2), 2000. https://doi.org/10.1590/S1516-44462000000600012

Brasil, Ministério da Saúde (2019). Relação Nacional de Medicamentos Essenciais 2020. Brasília, 217 p. FROM: https://bvsms.saude.gov.br/bvs/publicacoes/relacao_medicamentos_rename_2020.pdf

Brasil. Lei 8.080, de 19 de setembro de 1990. Dispõe sobre as condições para a promoção, proteção e recuperação da saúde, a organização e o funcionamento dos serviços correspondentes e dá outras providências. Diário Oficial da União, Brasília, DF, Seção 1, 19 set. 1990. FROM: https://www.planalto.gov.br/ccivil_03/leis//8080.htm. 
Brasil. Lei $\mathrm{n}^{0}$ 10.216, de 06 de abril de 2001. Dispõe sobre a proteção e os direitos das pessoas portadoras de transtornos mentais e redireciona o modelo assistencial em saúde mental. Diário Oficial da União, Poder Legislativo. Brasília, DF, Seção 1, Ed. 69, 9 abr. 2001, p. 2, 2001. https://legislacao.presidencia.gov.br/atos/?tipo=LEI\&numero=10216\&ano=2001\&ato=b4foXW E5kMNpWT0b8

Brasil. Organização Mundial da Saúde (OMS). Guia do Instrutor em Práticas da Boa Prescrição Médica. Departamento de Medicamentos Essenciais e Políticas de Medicamentos Genebra, Suíça, 2001.

From: https://www.paho.org/bra/index.php?option=com_docman\&view=download\&alias=806-guiado-instrutor-em-praticas-da-boa-prescricao-medica-6\&category_slug=vigilancia-sanitaria959\&Itemid $=965$

Brzozowski, F. S. \& Caponi, S. N. C. (2013). Medicalização dos Desvios de Comportamento na Infância: Aspectos Positivos e Negativos. Psicologia: Ciência e Profissão, 33 (1), 208-221. https://doi.org/10.1590/S1414-98932013000100016

Conrad, P. The medicalization of society. On the transformation of human conditions into treatable disorders. Baltimore: Johns Hopkins University Press; 2007.E-book. FROM: https://books.google.com.br/books?id=cAE5hIP5YkAC\&printsec=frontcover\&hl=ptBR\&source= gbs_ge_summary_r\&cad=0\#v=onepage \&q\&f=false

Couto, M. C. V.; Duarte, C. S.; Delgado, P. G. G (2008). A saúde mental infantil na Saúde Pública brasileira: situação atual e desafios. Rev. Bras. Psiquiatr., São Paulo, 30 (4), p.384-389. https://doi.org/10.1590/S1516-44462008000400015

Dalgalarrondo, P. (2018). Psicopatologia e semiologia dos transtornos mentais. Porto Alegre: ArtMed. E-book. From: https://integrada.minhabiblioteca.com.br/books/9788582715062.

Leitão, I. B.; Tristão, A. B. D. K. G.; Ronchi, J. P.; Avellar, L. Z. (2020, jan 31). Dez anos de um CAPSi: comparação da caracterização de usuários atendidos. Psicologia USP, 31, p. 1-14. https://doi.org/10.1590/0103-6564e190011

Ministério da Saúde. Portaria GM/MS no 336, de 19 de Fevereiro de 2002. Estabelece que os Centros de Atenção Psicossocial poderão constituir-se nas seguintes modalidades de serviços: CAPS I, CAPS II e CAPS III. Diário Oficial União, Brasília, DF, 9 fev. 2002. FROM :https://bvsms.saude.gov.br/bvs/saudelegis/gm/2002/prt0336_19_02_2002.html .

Ministério da Saúde. Secretaria de Atenção à Saúde. Departamento de Ações Programáticas Estratégicas. Caminhos para uma política de saúde mental infanto-juvenil/ Ministério da Saúde, Secretaria de Atenção à Saúde, Departamento de Ações Programáticas Estratégicas. - 2. ed. rev. - Brasília: Editora do Ministério da Saúde, 2005. http://www.ccs.saude.gov.br/saude_mental/pdf/sm_sus.pdf

Ministério da Saúde. Secretaria de Atenção à Saúde. Departamento de Atenção Especializada e Temática. Centros de Atenção Psicossocial e Unidades de Acolhimento como lugares da atenção psicossocial nos territórios: orientações para elaboração de projetos de construção, reforma e ampliação de CAPS e de UA / MS, Secretaria de Atenção à Saúde, Departamento de Atenção Especializada e Temática. - Brasília: MS, 2015.44 p.http://bvsms.saude.gov.br/bvs/publicacoes/centros_atencao_psicossocial_unidades_acolhi mento.pdf. 
Moreira, C. P., Torrenté, M. O. N. \& Jucá, V. J. S. (2018). Análise do processo de acolhimento em um Centro de Atenção Psicossocial Infantojuvenil: considerações de uma investigação etnográfica. Interface,22(67), p.1123-34. https://doi.org/10.1590/1807-57622017.0500

Ministério da Saúde. Secretaria de Atenção à Saúde. Departamento de Ações Programáticas Estratégicas. Saúde mental no SUS: os centros de atenção psicossocial / MS, Secretaria de Atenção à Saúde, Departamento de Ações Programáticas Estratégicas. - Brasília: MS, 2004b. 86 p.: il. color. - (Série F. Comunicação e Educação em Saúde) ISBN 85-334-0775-0. http://www.planalto.gov.br/ccivil_03/leis/leis_2001//10216.htm

OMS, Organização Mundial da Saúde (2018). Folha informativa: Depressão. FROM: https://www.paho.org/pt/topicos/depressao

OMS, Organização Mundial da Saúde (2018). Folha informativa: Suicídio. FROM: https://www.paho.org/pt/node/72968

Silva, A. (2017). Terapêutica farmacológica e complementar na perturbação do Espectro do Autismo: uma revisão. Dissertação (Mestrado)- Trabalho final mestrado integrado em medicina: Clínica universitária de Pediatria, Universidade de Lisboa, 49 pg. From: https://repositorio.ul.pt/bitstream/10451/30753/1/AnaDBSilva.pdf

Silva, S. N.; Lima, M. G.; Ruas, C. M. (2020). Uso de medicamentos nos centros de atenção psicossocial. Rev. Ciência \& Saúde Coletiva, 25 (7), p.2871-2882. DOI: 10.1590/141381232020257.23102018

Teixeira, E. H.; Jacinto, A.; Celeri, H. V.; Dalgalarrondo, P. (2013). Atypical antipsychotics in the treatment of pathological aggression in children and adolescents: literature review and clinical recommendations. Rev. Trends Psychiatry Psychother, 35 (3). https://doi.org/10.1590/S223760892013000300002

WHO, World Health Organization. Young People's Health - a Challenge for Society. Report WHO Study Group on Young People and Health for All. Technical Report Series 731. Geneva: WHO, 1986.

WORLD HEALTH ORGANIZATION (1985). Medicines: rational use of medicines. Fact sheet num. 338. May. From: http:// www.who.int/mediacentre/factsheets/fs338/en/ print.html.

Yamauti, S. M.; Bonfim, J. R. A.; Filho, S. B.; Lopes, L. C. (2017). Essencialidade e racionalidade da relação nacional de medicamentos essenciais do Brasil. Revista Ciência \& Saúde Coletiva, 22 (3), p. 975-986. http://dx.doi.org/10.1590/1413-81232017223.07742016. 